\title{
Asentamientos precarios de Costa Rica: impacto social y ambiental en proyectos de renovación urbana
}

\author{
David Aguilar Zúñiga \\ Maestría en Manejo de Recursos Naturales, Universidad Estatal a Distancia, 2050 San José, Costa Rica; daguilarz@gmail.com
}

Recibido 31-V-2017 • Corregido 04-IX-2017 • Aceptado 19-IX-2017

\begin{abstract}
Social and environmental impact in construction projects of urban renewal in Costa Rican slums. There are more than 189 slums in Costa Rica Metropolitan Area. The National Financial System for Housing has the eradication slums program. This financial model invests economic resources on those kind of settlements to improve infrastructure condition through construction processes. For this research, three slums assisted by PEPT were chosen to assess environmental and social conditions: Los Cuadros, Tirrases and Miramar. These settlements include five public spaces destined for parks, recreation, sports and urban areas, as well as a controlling space that is not managed by PEPT located in Los Cuadros. Environmental and social conditions were evaluated before and after the economic intervention to analyze indicators variation associated with water, soil, air, urban elements, waste, society and communal organization. A qualification system was created with similar structure used by the National Environmental Technical Secretariat (SETENA due to its acronym in Spanish) to evaluate the influence on construction projects. Four of five public spaces showed environmental improvements related to urban elements, lighting, water management, floor covering and waste management, and one of them showed social improvements related to occupants practices and customs. They are statistically significant after resources investment. The controlling space did not show representative statistical changes in the environmental characteristics assessed. Finally, the economic investment in public spaces located in slums offers not only social but environmental benefits.
\end{abstract}

Key words: Slums, precarious settlements, environmental impact, social impact, neighborhood improvement, urban development, Costa Rica.

En el mundo más de 800 millones de personas habitan en condiciones de pobreza (PNUD, 2015), un segmento de esta población se ubica dentro de los centros urbanos en asentamientos precarios. Estos lugares se caracterizan por presentar condiciones insalubres, carencia de servicios básicos, inadecuado manejo de aguas servidas, densificación, uso del suelo descontrolado, tenencia ilegal de los terrenos y exclusión social (UN-HABITAT, 2003).
RESUMEN: En el Gran Área Metropolitana de Costa Rica hay más de 189 asentamientos precarios. El Sistema Financiero Nacional para la Vivienda cuenta con el Programa de Erradicación de Asentamientos en Condición de Precario y Tugurio (PEPT). Este modelo financiero invierte recursos económicos en precarios para mejorar las condiciones de infraestructura, a través de procesos de construcción. Para esta investigación, se eligieron tres asentamientos precarios: Los Cuadros, Tirrases y Miramar, asistidos por el PEPT para evaluar las condiciones ambientales y sociales. Los asentamientos incluyen un total de cinco espacios públicos destinados para parques, áreas recreativas, deportivas y de carácter urbano, además de un espacio de control fuera del alcance del PEPT que se encuentra localizado en Los Cuadros. Se evaluaron las condiciones ambientales y sociales antes y después de la intervención económica, para analizar la variación de indicadores asociados a condiciones de agua, suelos, aire, mobiliario urbano, residuos, sociedad y organización comunal. Se confeccionó un sistema de calificación cualitativa con estructura similar al utilizado por la Secretaría Técnica Nacional Ambiental (SETENA) para la evaluación de impacto en proyectos de construcción. En cuatro de los cinco espacios públicos hubo mejoras ambientales, relacionadas con el mobiliario urbano, iluminación, manejo de aguas, recubrimiento de los suelos y manejo de residuos, y en uno de ellos hubo mejoras sociales relacionadas con prácticas y costumbres de sus ocupantes, que resultan estadísticamente significativas después de realizada la inversión de recursos. En el espacio de control no se evidenciaron cambios estadísticos representativos en las características ambientales valoradas. Se demuestra que la inversión económica en espacios públicos ubicados en asentamientos precarios ofrece beneficios sociales y ambientales.

Palabras clave: Asentamientos precarios, impacto ambiental, impacto social, mejoramiento barrial, renovación urbana, Costa Rica.

Para enfrentar la problemática de la pobreza y los precarios en Costa Rica, se creó, en el año 1986, el Sistema Financiero Nacional para la Vivienda (SFNV), junto con otros programas sociales. Esto fue el inicio de las funciones en trámite y construcción de vivienda de interés social, así como tareas propias de investigación y de definición de prioridades. A partir de ahí, se determinó que solo en el Gran Área Metropolitana para el año 1987, 
existían cerca de 104 asentamientos precarios, cifra que aumentó en un 82\% (189 asentamientos precarios) en un lapso de 25 años (CNPU, 2013).

Dentro de la intervención estatal auspiciada por el SFNV, se creó el Programa de Erradicación de Asentamientos en Condición de Precario y Tugurio (PEPT), modelo de gestión y desarrollo para atender los habitantes expuestos a pobreza y pobreza extrema, con déficit en vivienda e infraestructura, en búsqueda de condiciones que garanticen a sus ocupantes una adecuada calidad de vida (PE, 2008). El PEPT plantea resolver los problemas de infraestructura en su lugar de origen, a través de procesos de desarrollo social y construcción de obras civiles.

La Secretaría Técnica Nacional Ambiental (SETENA), entre otras de sus múltiples funciones, fiscaliza las evaluaciones ambientales ligadas a los proyectos de construcción de interés social. Esto se realiza con herramientas que evalúan su impacto ambiental (CNPU, 2013), donde las técnicas ya estandarizadas que se aplican no permiten una cuantificación del cambio suscitado en proyectos de erradicación de precarios y de mejoramiento barrial.

Los asentamientos precarios evaden la normativa técnica y legal aplicable, dado que su conformación es espontánea, descontrolada, donde no media ningún tipo de planificación, de modo que desde su inicio a su consolidación se propicia y evidencia el daño ambiental (Chacón \& Freer, 1999).

En vista de lo anterior, todos aquellos reglamentos que establece el Gobierno Central y las municipalidades, así como cualquier otro mecanismo técnico aplicable, incluidos los de SETENA, se omiten y se desestiman, lo que ocasiona afectaciones ambientales y sociales en la zona de consolidación, así como en áreas vecinas. Para valorar si las acciones gubernamentales en los proyectos financiados por el PEPT producen mejoras en las condiciones ambientales y sociales, es necesario medir, evaluar y realizar comprobaciones ligadas a los mecanismos de cambio utilizados. En este caso, la transformación del espacio por medio de obras de construcción y el abordaje social complementario.

\section{MÉTODOS}

Esta investigación evalúa diferentes componentes con capacidad de modificar las características ambientales y sociales del espacio, la evaluación ejecutada es similar a la que emplea SETENA en sus formularios para la valoración de impacto, según se indica en el Decreto 32712-MINAE del Gobierno de Costa Rica (MINAE, 2005), cuyo alcance se enfoca en estimar cambios en los ámbitos de aire, agua, suelo, entre otros, de los diferentes proyectos de construcción que se tramiten en el país.

La valoración ambiental y social se aplicó en tres proyectos con disponibilidad presupuestaria para su ejecución en el año 2014 y 2015, con enfoque en el mejoramiento del espacio público y que perteneciesen a la Región Central de Costa Rica. Los proyectos evaluados son: Los Cuadros, Tirrases y Miramar, que contienen un total de cinco espacios públicos, con distintos alcances en sus obras de construcción (Cuadro 1).

En el análisis se incluye una zona de control ubicada en el proyecto Los Cuadros. Este espacio no fue incluido dentro del PEPT, dado que otras instancias gubernamentales proyectaban otros planes de inversión. Durante la ejecución de las obras seleccionadas no se presentó ningún tipo de desarrollo en esta área control.

Las obras de construcción en los diferentes espacios incluyen obras de rehabilitación y equipamiento, entre

CUADRO 1

Características de los espacios de evaluación

\begin{tabular}{llccc}
\multicolumn{1}{c}{ Espacio Público } & \multicolumn{2}{c}{ Coordenadas } & Inversión (\$) & Área $\left(\mathrm{m}^{2}\right)$ \\
Los Cuadros, Goicoechea & Norte & Oeste & & \\
$\quad$ Parque CEN & & & & \\
$\quad$ Plaza de Deportes & $9^{\circ} 57^{\prime} 41^{\prime \prime}$ & $84^{\circ} 00^{\prime} 39^{\prime \prime}$ & 186306,28 & 1238,00 \\
$\quad 9^{\circ} 57^{\prime} 37^{\prime \prime}$ & $84^{\circ} 00^{\prime} 29^{\prime \prime}$ & 533859,45 & 10207,00 \\
$\quad 9^{\circ} 57^{\prime} 40^{\prime \prime}$ & $84^{\circ} 00^{\prime} 32^{\prime \prime}$ & 0,00 & 3596,00 \\
$\begin{array}{l}\text { Mirque Triángulo (Control) } \\
\quad \text { Infraestructura Residencial }\end{array}$ & $9^{\circ} 49^{\prime} 29^{\prime \prime}$ & $84^{\circ} 19^{\prime} 45^{\prime \prime}$ & 223166,24 & 1136,00 \\
$\begin{array}{l}\text { Tirrases, Curridabat } \\
\quad \text { Conjunto Pinos del Este }\end{array}$ & $9^{\circ} 54^{\prime} 05^{\prime \prime}$ & $84^{\circ} 01^{\prime} 38^{\prime \prime}$ & 90992,96 & 286,00 \\
$\quad$ Accesos Puente Peatonal Colonia Cruz & $9^{\circ} 54^{\prime} 08^{\prime \prime}$ & $84^{\circ} 01^{\prime} 27^{\prime \prime}$ & $34.959,64$ & 117,00 \\
\hline
\end{tabular}


las más comunes se encuentran: aceras, mobiliario urbano, alumbrado público, sistemas de evacuación pluvial, superficies de concreto y arborización (Cuadro 2).

En cada espacio público, se realizaron dos mediciones, antes y después de la etapa constructiva, a partir de la observación estructurada in situ, para aquellos indicadores de carácter ambiental, asociados a condiciones de calidad del aire, conducción de aguas, afectaciones al suelo, manejo de residuos, biodiversidad y mobiliario urbano.

Las variables anteriores son algunas de las que usualmente se modifican en las obras de construcción de infraestructura, dado que afectan directamente la calidad de vida de sus habitantes, además de que facilitan su interacción con el espacio y con otros individuos. De igual forma, este grupo de variables son similares a las que examina SETENA en sus formularios de impacto ambiental.

El análisis social se efectuó por medio de talleres participativos, compuestos por al menos diez integrantes de la comunidad, quienes participaron en ambas etapas de la calificación (antes-después). En estos talleres, se discutieron las variables ambientales y sociales, estas últimas relacionadas con condiciones de seguridad social, uso del espacio, organización comunitaria, entre otras. Posteriormente, el grupo participante -de forma consensuada- asignó valores según la escala propuesta, para establecer los valores finales que luego fueron verificados en la herramienta de control estadístico.

Se estableció un sistema de calificación donde el valor menor corresponde a la condición menos favorable, en una escala de tipo ordinal. La escala es diferente para cada indicador, donde el menor valor posible es uno.
Para asignar los valores en la escala ordinal, se utilizaron relaciones de áreas (área en estudio dividida entre el área total del espacio) medidas acordes a los planos de construcción o levantamientos topográficos, o bien por observación de existencia o ausencia de los elementos en evaluación.

Para comprobar si el PEPT produce cambios favorables en las características sociales y ambientales, se aplicó la prueba estadística de Wilcoxon (rangos con signo). Esta prueba se realizó de acuerdo a una valoración total combinada por indicadores, así como seccionada por grupo. De forma adicional, se determinó la variación porcentual por cada indicador y se ilustró en gráficos radiales.

Para Los Cuadros y Miramar, se efectuó la valoración ambiental y social, de tal forma que se realizó un análisis combinado y separado de cada agrupación de indicadores (Cuadro 3). Se aclara que para Tirrases no se procedió con la valoración social, dado que no se identificó ninguna agrupación u organización comunitaria establecida para el desarrollo del proyecto de construcción, por esta carencia no se aplicó el mismo procedimiento de valoración utilizado en los otros dos proyectos.

\section{RESULTADOS}

Según el análisis estadístico, se interpreta que solamente el espacio denominado Conjunto Pinos del Este de Tirrases no presentó un cambio significativo en sus indicadores ambientales (Cuadro 4). En el caso de la valoración social, tanto la plaza de deportes de Los Cuadros

CUADRO 2

Alcance de las obras de construcción

Espacio público

Parque CEN - Los Cuadros

Plaza de Deportes - Los Cuadros

Miramar - Infraestructura Residencial

Tirrases - Conjunto Pinos del Este

Tirrases - Accesos Puente Peatonal Colonia Cruz

\section{Descripción de obras de construcción}

Construcción de parque en lote baldío, con inclusión de aceras, elementos de desfogue pluvial, sistema de iluminación, mobiliario urbano, elementos de tránsito peatonal y vehicular, además de áreas verdes.

Renovación y remodelación de elementos existentes de tránsito peatonal y deportivo. Construcción de juegos infantiles, parque skate y mobiliario urbano, además de elementos de infraestructura.

Construcción de obras de infraestructura para el manejo de aguas, distribución de agua potable y tránsito vehicular - peatonal.

Construcción de corredor techado para acceso al transporte público. Conecta, además, con espacios públicos y viviendas.

Construcción de obras de infraestructura circundantes al puente peatonal existente. Lo anterior incluye elementos de tránsito peatonal, manejo de aguas e iluminación. 
CUADRO 3

Descripción de análisis realizado por espacio

\begin{tabular}{lccc}
\multicolumn{1}{c}{ Espacio } & Análisis Ambiental & Análisis Social & Análisis Combinado \\
Los Cuadros - Parque CEN & Sí & Sí & Sí \\
Los Cuadros - Plaza de Deportes & Sí & Sí & Sí \\
Miramar - Infraestructura Residencial & Sí & Sí & Sí \\
Tirrases - Conjunto Pinos del Este & Sí & No & No \\
Tirrases - Accesos Puente Peatonal Colonia Cruz & Sí & No & No \\
\hline
\end{tabular}

CUADRO 4

Análisis Combinado: Indicadores ambientales y sociales

\begin{tabular}{|c|c|c|c|c|c|}
\hline & $\begin{array}{l}\text { Los Cuadros - } \\
\text { Parque CEN }\end{array}$ & $\begin{array}{l}\text { Los Cuadros - } \\
\text { Plaza de Deportes }\end{array}$ & $\begin{array}{l}\text { Miramar - Infraestructura } \\
\text { Residencial }\end{array}$ & $\begin{array}{l}\text { Tirrases - Conjunto } \\
\text { Pinos del Este }\end{array}$ & $\begin{array}{l}\text { Tirrases - Accesos Puente } \\
\text { Peatonal Colonia Cruz }\end{array}$ \\
\hline Z & $-3,624^{b}$ & $-2,651^{b}$ & $-2,636^{b}$ & & \\
\hline Sig. asintót. (bilateral) & 0,000 & 0,008 & 0,008 & N/A & N/A \\
\hline \multicolumn{6}{|c|}{ Análisis para indicadores ambientales } \\
\hline Z & $-2,763^{b}$ & $-2,700^{b}$ & $-2,299^{b}$ & $-1,466^{b}$ & $-2,060^{b}$ \\
\hline Sig. asintót. (bilateral) & 0,006 & 0,007 & 0,022 & 0,143 & 0,039 \\
\hline \multicolumn{6}{|c|}{ Análisis para indicadores sociales } \\
\hline Z & $-2,588^{b}$ & $0,000^{c}$ & $-1,342^{b}$ & & \\
\hline Sig. asintót. (bilateral) & 0,010 & 1,000 & 0,180 & N/A & $\mathrm{N} / \mathrm{A}$ \\
\hline \multicolumn{6}{|c|}{ Análisis Ambiental: Espacio de Control, Los Cuadros } \\
\hline Z & & & -0.577 & & \\
\hline Sig. asintót. (bilateral) & & & 0.5637 & & \\
\hline
\end{tabular}

como la infraestructura residencial de Miramar no presentaron un cambio significativo (Cuadro 4).

El espacio de control ubicado en Los Cuadros otorga un valor de $Z$ de $-0,577$ y un valor de $p$ de 0,5637 , por tanto, se evidencia que no hay un cambio significativo entre ambas mediciones (Cuadro 4).

Para el espacio de control se observa que las variables ambientales no modificaron su calificación de acuerdo a la trasposición de áreas (Fig. 1a), a excepción del material particulado y el manejo de residuos sólidos, en donde se presentó una mejora de su condición. Se evidencia, además, una alteración no favorable según la escala nominal asignada en la línea de vegetación.

Las valoraciones ambientales para los cinco espacios públicos muestran áreas de cobertura iguales o mayores a las obtenidas inicialmente (Fig. 1b a 1f). Algunos de los indicadores presentaron reducciones que se interpretan como condiciones no favorables en su desarrollo; por ejemplo, la reducción de vegetación en el Parque CEN de Los Cuadros (Fig. 2) y el manejo de escombros en el espacio Conjunto Pinos del Este de Tirrases (Fig. 1e).
Las variabilidades sociales en los tres espacios públicos de dos de los asentamientos intervenidos muestran que solo en el Parque CEN de Los Cuadros hubo un incremento favorable de varios parámetros respecto de sus condiciones iniciales (Fig. 2a). Para los otros dos espacios, se evidencia que solo mejoraron las condiciones de paisaje (Fig. 2b y 2c), y de salubridad para el caso de la infraestructura residencial de Miramar (Fig. 2c).

La variación porcentual (Cuadros 5 y 6) señala el valor resultante para cada uno de los indicadores observados. En el ámbito ambiental, dos indicadores otorgaron valores negativos (escorrentía y vegetación), los cuales se interpretan como una disminución en su calidad respecto a su condición inicial. En el área social no se evidencian resultados negativos, sin embargo, algunas de sus condiciones no presentan mejoras perceptibles correspondientes a los ámbitos de seguridad y organización comunal.

Para el caso de los proyectos Los Cuadros y Miramar, el puntaje descrito (Cuadro 7) combina lo obtenido en la valoración ambiental y social, para los dos tiempos determinados (antes-después). En el escenario del proyecto 


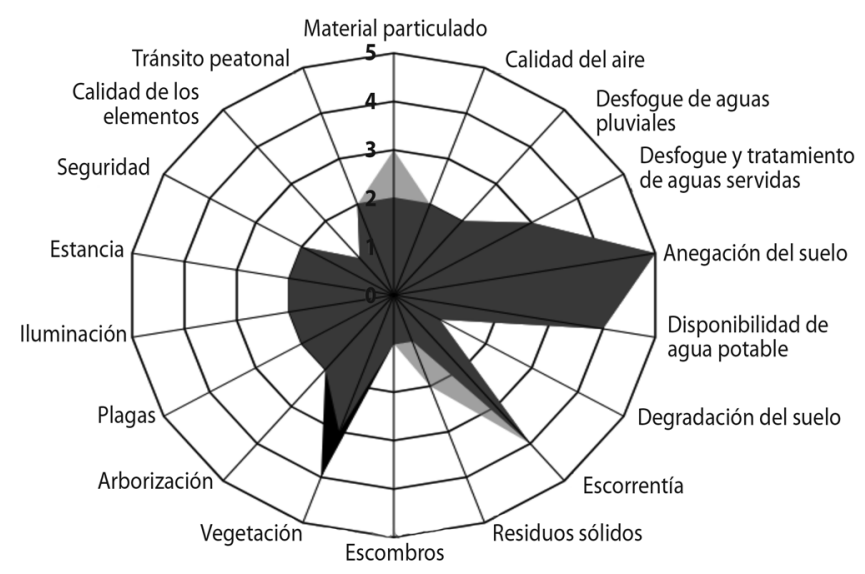

Calificación antes $\square$ Calificación después

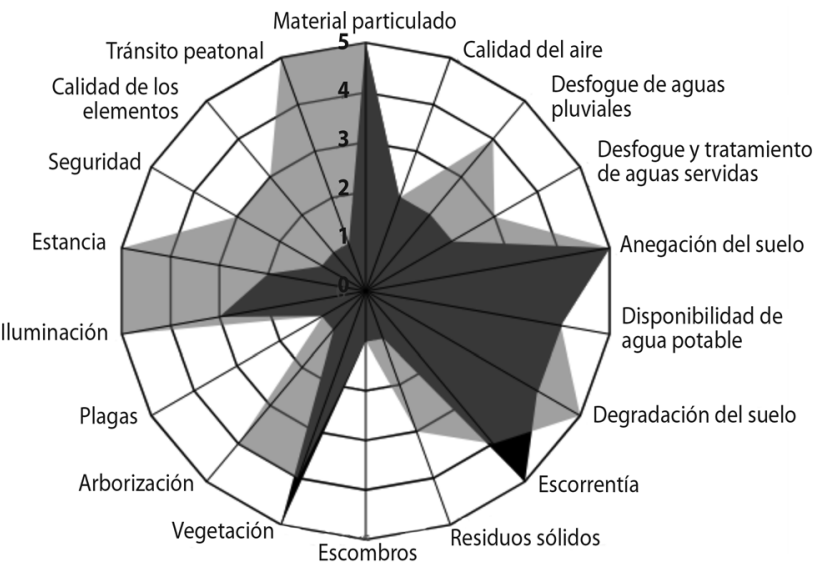

Calificación antes $\square$ Calificación después

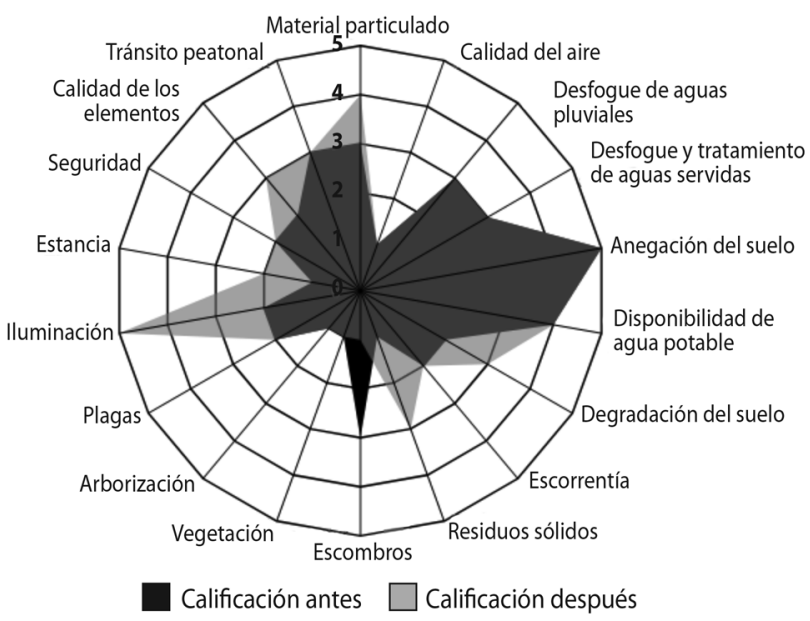

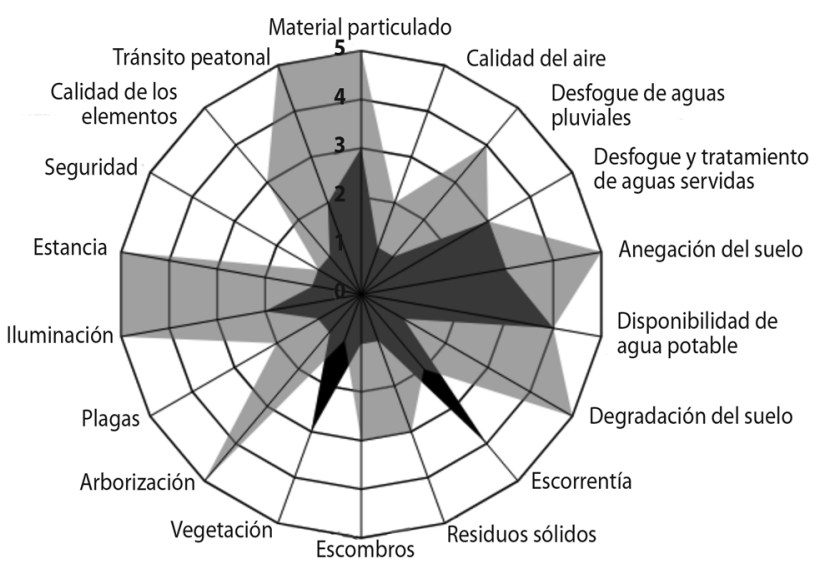

Calificación antes $\square$ Calificación después

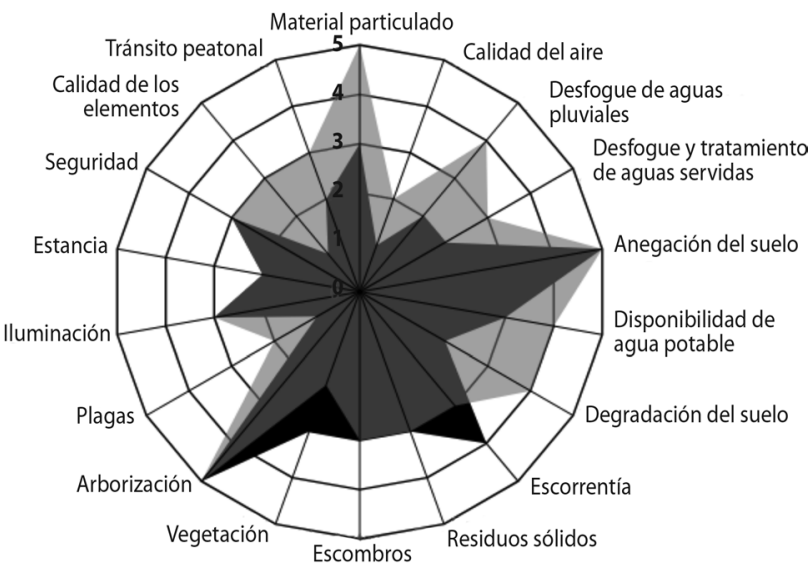

Calificación antes $\square$ Calificación después

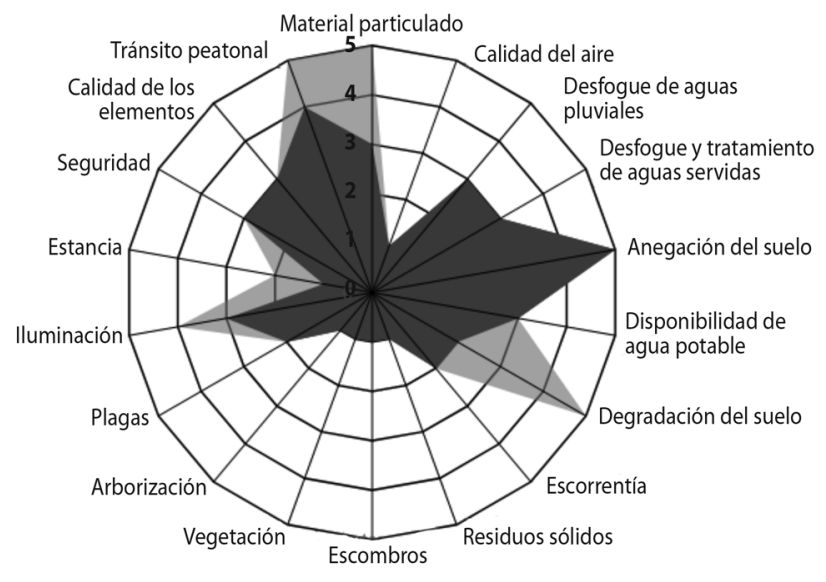

Calificación antes $\square$ Calificación después

Fig. 1. Valoración ambiental. A. Control - Los Cuadros. B. Parque CEN - Los Cuadros. C. Plaza de Deportes - Los Cuadros. D. Conjunto Pinos del Este - Tirrases. E. Conjunto Pinos del Este - Tirrases. F. Accesos Puente Peatonal Colonia Cruz-Tirrases. 

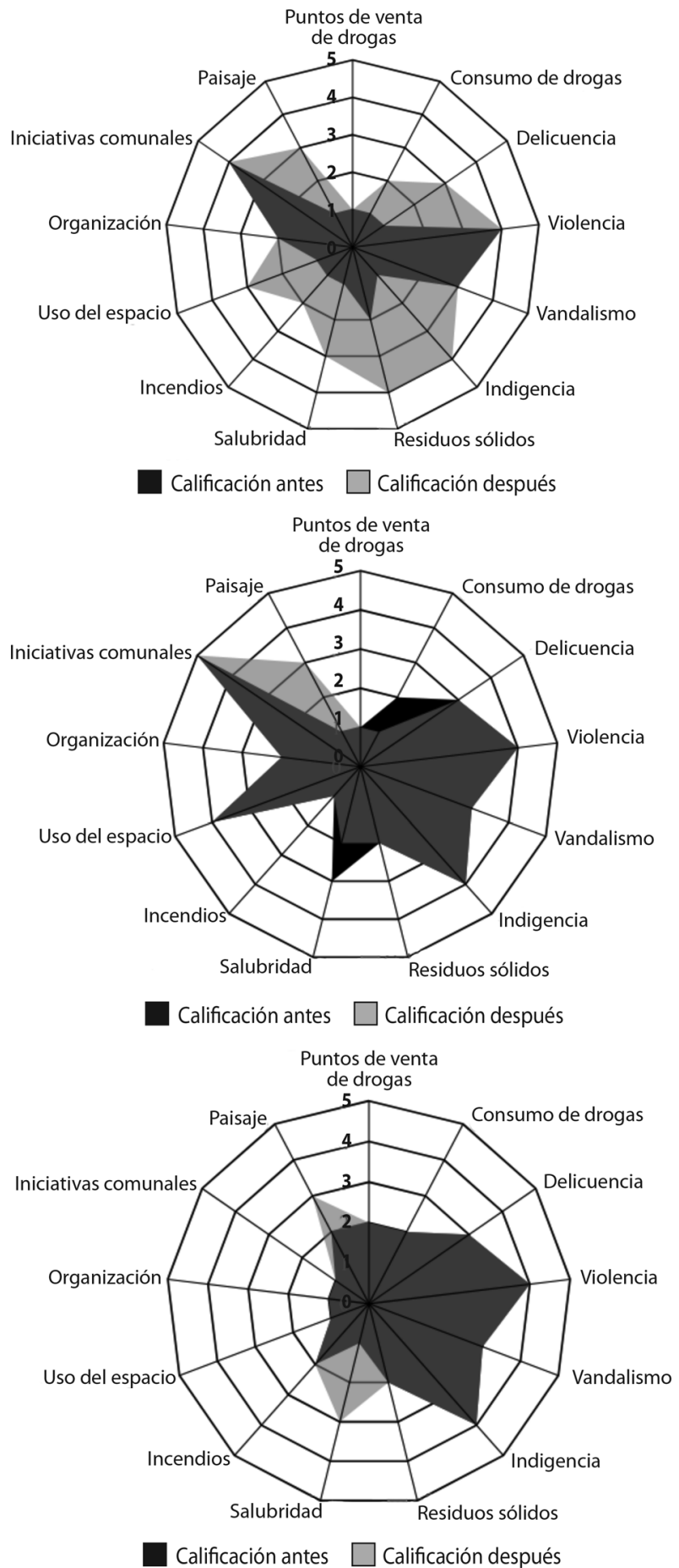

Fig. 2. Valoración social. A. Parque CEN - Los Cuadros. B. Plaza de Deportes - Los Cuadros. C. Infraestructura Residencial - Miramar.
Tirrases la calificación se estima en razón del puntaje máximo del análisis ambiental, dado que en este no se realizó la valoración de características sociales.

Del análisis grupal que combina indicadores ambientales y sociales para los proyectos Los Cuadros y Miramar, se obtiene que hay un cambio significativo demostrado estadísticamente entre la condición inicial y la condición final. Este cambio se cuantifica de forma de variación porcentual en cada sitio, el Parque CEN presenta el mayor valor de cambio (38,60\%), seguido por la plaza de deportes $(17,54 \%) y$, por último, la infraestructura de Miramar (12,28\%). Resalta, además, que estos espacios recibieron la mayor cantidad de recursos económicos (Cuadro 1).

Para el espacio Conjunto Pinos del Este de Tirrases, se obtiene que la inversión no produjo mejoras ambientales según la comprobación estadística. Sin embargo, la variación porcentual indica un aumento del 9,72\% respecto de su condición original. Lo anterior se demuestra de forma cualitativa al contar con más elementos de iluminación, inclusión de elementos de estancia y de mejor calidad a los que se encontraron previamente.

La variación porcentual anterior puede ser visualizada en los gráficos radiales indicados previamente. Si la huella de la fase final (color gris) es mayor a la señalada en la fase inicial de medición (color negro), se interpreta como variación positiva, en caso de que ambas huellas sean similares la prueba estadística identifica que no hubo un cambio representativo, tal y como sucede en el espacio Conjunto Pinos del Este para la valoración ambiental. Lo anterior también aplica para la valoración social de la Plaza de Deportes de Los Cuadros y la Infraestructura de Miramar.

En la valoración social se obtiene que para la plaza de deportes de Los Cuadros no hay cambios significativos. Según la percepción comunal el espacio es seguro. Además, los participantes del taller participativo consideran que mantener la limpieza de la zona se dificultó por el aumento en su ocupación y tenencia. El grupo expone un reforzamiento en la cohesión social y la apropiación de este espacio, pero no fue incidente en la creación de nuevas organizaciones comunales.

En el mismo análisis de valoración social, la infraestructura residencial de Miramar resalta la mejora del paisaje y de la salubridad del sitio. Sin embargo, el proyecto de bono comunal no promovió la creación de nuevas estructuras comunales.

En el análisis individual por indicador, se observa que para la valoración por escorrentía y vegetación hay cambios desfavorables. El cambio en la escorrentía se debe a que la superficie del suelo de los diferentes espacios fue impermeabilizada parcialmente, lo que ocasionó un 
CUADRO 5

Variación porcentual de indicadores ambientales para todos los espacios de estudio

\begin{tabular}{|c|c|}
\hline \multicolumn{2}{|l|}{$\begin{array}{l}\text { Indicadores Ambientales } \\
\text { Material particulado }\end{array}$} \\
\hline $\begin{array}{l}\text { Material particulado } \\
\text { Calidad del aire } \\
\text { Desfogue de aguas pluviales } \\
\text { Desfogue y tratamiento de aguas ser } \\
\text { Anegación del suelo } \\
\text { Disponibilidad de agua potable } \\
\text { Degradación del suelo } \\
\text { Escorrentía } \\
\text { Residuos sólidos } \\
\text { Escombros } \\
\text { Vegetación } \\
\text { Arborización } \\
\text { Plagas } \\
\text { lluminación } \\
\text { Estancia } \\
\text { Seguridad } \\
\text { Calidad de los elementos } \\
\text { Tránsito peatonal }\end{array}$ & \\
\hline $\begin{array}{r}\text { CUADRO } 6 \\
\text { Variación porcentual de indi } \\
\text { para Los Cuadros y }\end{array}$ & ociales \\
\hline Indicadores sociales & Variación \% \\
\hline Puntos de venta de drogas & 0,00 \\
\hline Consumo de drogas & 0,00 \\
\hline Delincuencia & 22,22 \\
\hline Violencia & 0,00 \\
\hline Vandalismo & 0,00 \\
\hline Indigencia & 25,00 \\
\hline Residuos sólidos & 25,00 \\
\hline Salubridad & 37,50 \\
\hline Incendios & 20,00 \\
\hline Uso del espacio & 25,00 \\
\hline Organización & 0,00 \\
\hline Iniciativas comunales & 0,00 \\
\hline Paisaje & 55,56 \\
\hline
\end{tabular}

Variación \% Espacio de Control

$\begin{array}{cc}29,17 & 33,33 \\ 25,00 & 0,00 \\ 38,89 & 0,00 \\ 13,33 & 0,00 \\ 8,00 & 0,00 \\ 5,26 & 0,00 \\ 50,00 & 0,00 \\ -30,77 & 0,00 \\ 46,15 & 50,00 \\ 0,00 & 0,00 \\ -44,44 & -33,33 \\ 43,75 & 0,00 \\ 22,22 & 0,00 \\ 40,91 & 0,00 \\ 56,25 & 0,00 \\ 16,67 & 0,00 \\ 46,67 & 0,00 \\ 42,86 & 0,00\end{array}$

aumento en los caudales y en la descarga hidráulica a cuerpos receptores. Sin embargo, estas impermeabilizaciones por su parte ofrecen mejores superficies para el tránsito peatonal y el desarrollo de actividades recreativas. Por otra parte, al recubrir las superficies se elimina la vegetación natural que crece en lotes baldíos, misma que no fue sustituida por otro tipo de especies como forma de compensación, por ejemplo, jardineras con arbustos o parches vegetales.

Las condiciones ambientales que obtuvieron mayor incremento y que, por tanto, prueban lo favorable de la inversión económica estatal, inciden en la disminución de la degradación de los suelos y los residuos sólidos dispersos, el aumento en la arborización y la mejora de los elementos de iluminación. Este último propicia mayor confianza en la ocupación del espacio, incrementa sus horas de utilización y mejora el tránsito peatonal.

CUADRO 7

Calificación máxima por espacio público

\begin{tabular}{lccc}
\multicolumn{1}{c}{ Espacio Público } & \multicolumn{2}{c}{ Calificación (\%) } & Variación (\%) \\
& Antes & Después & 0,88 \\
Los Cuadros - Control & 36,84 & 37,72 & 38,60 \\
Los Cuadros - Parque CEN & 50,00 & 88,60 & 17,54 \\
Los Cuadros - Plaza de Deportes & 71,05 & 88,60 & 12,28 \\
Miramar - Infraestructura & 66,67 & 78,95 & 9,72 \\
Tirrases - Conjunto Pinos del Este & 56,94 & 66,67 & 11,11 \\
Tirrases - Accesos Puente Peatonal & 58,33 & 69,44 & \\
\hline
\end{tabular}


En el ámbito social, la percepción de los habitantes resalta la disminución de los residuos, lo que influye directamente en aspectos de salubridad y ornato, que resultan finalmente en una optimización de la calidad del paisaje.

A nivel general, se contabiliza un balance positivo en la mejora de las condiciones ambientales, y se evidencia algunos beneficios sociales de los espacios intervenidos. Se concluye, además, que los recursos enfocados a zonas deportivas y recreativas fueron los que se presentaron mayor cambio respecto a su condición original, así como mejor aceptación de parte de sus beneficiarios.

\section{DISCUSIÓN}

El aporte institucional en las políticas públicas infiere directamente en el desarrollo de los proyectos de mejoramiento barrial (Magalhaes \& Villarosa, 2012). Al desestimar la inversión económica propuesta para el espacio de control ubicado en Los Cuadros, no se registró ningún tipo de desarrollo en infraestructura y de carácter social una vez que este terreno fuera extraído del PEPT; en consecuencia, se obtiene un espacio ocioso y sin aprovechamiento comunal.

El compromiso, seguimiento y gestión de agentes gubernamentales y privados beneficia la transformación prolongada del espacio y su desarrollo (Magalhaes \& Villarosa, 2012), con resultados estadísticamente significativos y tangibles en los tres asentamientos precarios intervenidos. Resultados similares obtuvieron Campos, García, Larenas y Simo (2009) en Chile y Cataluña, Magalhaes y Villarosa (2012) en favelas de Brasil y Sánchez y Gutiérrez (2014) en Medellín, donde prosigue la evolución del espacio físico en nuevas dinámicas sociales, legales, económicas, culturales, deportivas y recreativas, ya que estas zonas transformadas mejoran la calidad de vida de sus ocupantes (UN-HABITAT, 2013).

La estancia en los espacios públicos asociada a aquellas características que permiten identificar su utilidad (Rangel, 2002) ofreció mayor variación en las zonas evaluadas, lo cual genera más inclusividad según sea el diseño (Segovia \& Jordán, 2005). Además, este esquema que es el utilizado en las diferentes etapas del desarrollo de los proyectos de bono comunal y del PEPT implicó una mejora en el tránsito peatonal, descanso, ocio, entre otros, se asocia al trabajo con equipos multidisciplinarios y con diferentes organizaciones comunales (Flores \& Gonzalez, 2007).

El paisaje, según la caracterización social, se vincula directamente con la condición de estancia ya mencionada.
Según el análisis social, el paisaje representó el mayor cambio positivo desde la perspectiva de los participantes. Estas variables influyen de manera fundamental en el reforzamiento del apego y la identidad comunal creada en un espacio público (Vidal \& Pol, 2005), y justifican en alguna medida la nula variación en iniciativas comunales al ser espacios consolidados.

Un grupo de indicadores ambientales mostraron un porcentaje de variación superior al 40\% (Cuadro 5); por su parte, el indicador salubridad de la evaluación social estuvo cerca de este valor. Dos de los elementos anteriores tienen una asociación directa con la apreciación de seguridad del espacio público, se ha identificado que aquellos lugares con buen mantenimiento estimulan de forma positiva la percepción de seguridad (Espinoza, Méndez \& Monge, 2017), y junto con sistemas eficientes de iluminación ofrecen a sus ocupantes un mayor control colectivo del espacio, además de que se amplía la oferta de actividades que en ellos se pueda realizar (UNHABITAT, 2013).

En el caso de la plaza de deportes del proyecto Los Cuadros, existe una discordancia con los principios expuestos por UN-HABITAT (2007), dado que el uso colectivo del espacio promovió en cierta medida el consumo de drogas y alcohol. Esto coincide con lo descrito por Díaz y Escárcega (2009) en las desventajas que lleva cualquier actividad en el desarrollo, además de que se heredan las preocupaciones culturales y sociales de estos espacios en las próximas generaciones, al mismo tiempo de que tales comportamientos reinciden en que el espacio público sea percibido como una amenaza (Segovia \& Jordán, 2005; Espinoza et al., 2017).

No obstante, la variación porcentual fue poco significativa en los ejes relacionados con los temas de seguridad a nivel general, a excepción del indicador de delincuencia en uno de los espacios públicos. Pese a lo anterior, la apropiación continua del espacio (Vidal \& Pol, 2005) en trabajo coordinado puede ser una herramienta fundamental para desvalorizar esta condición de carácter negativo.

La Directriz 027 (PE, 2008) enfatiza la atención de los sistemas hidráulicos de los asentamientos precarios, dado que el desfogue y tratamiento de aguas servidas, manejo de aguas pluviales y servicios de dotación de agua potable repercuten directamente en la calidad de vida de la población. En estos proyectos en particular, la inversión fue reducida a ciertos espacios públicos, de tal forma que los porcentajes en las categorías de aguas servidas y acueducto se consideren bajos; sin embargo, para Miramar de Puriscal, la atención de dichos sistemas 
fue fundamental, así como la principal preocupación de sus habitantes previo al proceso de intervención.

Para el manejo de aguas pluviales, se obtuvo una variación relativamente alta $y$, por ello, se redujeron los valores de permeabilidad de las capas superficiales $y$, en consecuencia, la anegación del suelo, lo que potencia el desarrollo sostenible de los asentamientos al resolver las deficiencias en los sistemas de conducción y tratamiento de estas aguas, tal y como lo señala Winchester (2006).

Al resolverse diferentes problemáticas de los espacios públicos, se reflejan aspectos colaterales que traen nuevos beneficios, entre ellos la reducción de malos olores por desagües y residuos, se merma la proliferación de plagas y se restringen prácticas nocivas como la quema de residuos y la utilización del espacio público como vertedero de escombros.

La vegetación urbana es un aspecto de gran influencia para recuperar el espacio público (Antolín, 2010); sin embargo, su inclusión debe responder a un diseño (Domínguez, Jungmann, Miranda, Vargas, Irarrázabal \& Peña, 2009) y a condiciones autóctonas que garanticen su preservación. Esta condición se vio afectada en los espacios atendidos, debido a la construcción de superficies pavimentadas, pero necesarias para el aprovechamiento del lugar, de modo que se recomienda la implementación de soluciones alternativas como jardines verticales y arborización robusta que sobrelleve la ocupación continua de los espacios públicos.

Los resultados exhiben los beneficios del PEPT y se estimula la revisión de los criterios de diseño de estos espacios públicos, de tal forma que pueda obtenerse mayores variaciones a nivel socio-ambiental y se potencie la utilización de los recursos financieros del Estado costarricense. De manera complementaria, corresponde investigar directamente otras repercusiones de estos proyectos en materia de salud, sustentabilidad, cohesión social y rentabilidad económica, en aras de una buena inversión.

\section{AGRADECIMIENTOS}

A la Fundación Costa Rica Canadá, por ofrecer las condiciones necesarias para desarrollar este proyecto de investigación. A los profesionales de la Universidad Estatal a Distancia: Zaidett Barrientos, Martha Marín, Sonia Avendaño, Javier Rodriguez y Ligia Bermúdez.

El artículo integra el tema de tesis del candidato al grado de Master Scientiae en la Maestría de Manejo de Recursos Naturales con énfasis en Gestión Ambiental de la Universidad Estatal a Distancia de Costa Rica.

\section{REFERENCIAS}

Antolín, J. C. (2010). Recuperación de áreas verdes, educación social incluyente. Hekademus: Revista Científica de la Fundación Iberoamericana para la Excelencia Educativa, 3(7), 45-63. Recuperado de: http://hekademus.calidadpp.com/numeros/07/Hekademus_07_06.pdf

Campos, F., García, L., Larenas, J., \& Simó, M. (2009). Regeneración urbana en Chile y Cataluña. Cuadernos de vivienda y urbanismo, 2(4), 186 - 208.

Chacón, L., \& Freer, E. (1999). El precarismo: análisis histórico y su desarrollo en el distrito de Pavas, San José, Costa Rica. Revista Costarricense de Ciencias Médicas, 20 (3-4), 195-213. Recuperado de: http:// www.scielo.sa.cr/scielo.php?script=sci_arttext\&pid=S0253-29481999000200007\&lng=es\&nrm=iso\&tln$\mathrm{g}=\mathrm{es}$

CNPU, Consejo Nacional de Planificación Urbana Costa Rica. (2013). PlanGAM 2013 Plan Nacional de Desarrollo Urbano para la Gran Área Metropolitana 2013. Recuperado de https://www.mivah.go.cr/Biblioteca_PlanGAM.shtml

Díaz, R., \& Escárcega, S. (2009). Desarrollo sustentable. Oportunidad para la vida. México: McGraw-Hill.

Domínguez, J. I., Jungmann, R., Miranda, M., Vargas, A., Irarrázabal, R., \& Peña, R. (2009). Forestación urbana, una alternativa real para combatir la contaminación ambiental. Camino al Bicentenario, Propuestas para Chile, Concurso Políticas Públicas 2009. Recuperado de: http://www.munitel.cl/Documentos_de_Interes/ Propuesta_Forestacion_Urbana_Concurso_Politicas_ Publicas_2009_UC.pdf

Espinoza, J., Méndez, V. H., \& Monge, J. (2017). Percepción de seguridad, uso y mantenimiento de los parques municipales en Costa Rica, según el sexo de los usuarios. Cuadernos de Investigación UNED, 9(1), 65 - 71. doi: 10.22458/urj.v9i1.1680

Flores, R., \& González, M. (2007). Consideraciones sociales en el diseño y planificación de parques urbanos. Economía, Sociedad y Territorio, 6(24), 913-951. Recuperado de: http://www.redalyc.org/articulo.oa?id=11162403

Magalhaes, F., \& Villarosa, F. (2012). Slum Upgrading: Lessons learned from Brazil. Inter - American Development Bank.

MINAE, Ministerio de Ambiente y Energía Costa Rica. (18 de enero de 2005). Decreto No. 32712-MINAE Manual de Instrumentos Técnicos para el Proceso de Evaluación de Impacto Ambiental (Manual de EIA) - Parte II. Diario La Gaceta, 223. Recuperado de http://www.setena.go.cr/ formularios.html

PE, Poder Ejecutivo de Costa Rica. (08 de mayo de 2008). Directriz: 027 Bono colectivo para obras de infraestructura en asentamientos precarios, programa de erradicación de tugurios. Diario La Gaceta, 88. Recuperado de: http://www.pgrweb.go.cr/scij/Busqueda/Normativa/ Normas/nrm_norma.aspx?param1=NRM\&nValor1=1\&nValor $2=63058 \&$ nValor3=72281\&strTipM $=F N$ 
PNUD, Programa de las Naciones Unidas para el Desarrollo. (2015). Informe sobre Desarrollo Humano 2015. Recuperado de http://www.undp.org/content/undp/ es/home/librarypage/hdr/2015-human-development-report.html

Rangel, M. (2002). La recuperación del espacio público para la sociabilidad ciudadana. [Congreso Internacional del Medio Ambiente y Desarrollo Sustentable 2002]. Recuperado de: http://www.saber.ula.ve/bitstream/123456789/13458/1/recuperacion_spacio.pdf

Sánchez, L., \& Gutiérrez, A. (2014). Potencialidades de la participación en la construcción de ciudad desde intervenciones urbanas en asentamientos precarios. América Latina Hoy, 68, 119 - 136. doi: 10.14201/alh201468119136

Segovia, O., \& Jordán, R. (2005). Espacios públicos urbanos, pobreza y construcción social. Serie Medio ambiente y desarrollo. Recuperado de: http://repositorio.cepal.org/bitstream/handle/11362/5660/ S0501090_es.pdf?sequence $=1$
UN-HABITAT, Programa de las Naciones Unidas para los Asentamientos Humanos. (2013). Activaciones urbanas para la apropiación del espacio público. Recuperado de: https://unhabitat.org/books/activaciones-urbanas-para-la-apropiacion-del-espacio-publico/

UN-HABITAT, Programa de las Naciones Unidas para los Asentamientos Humanos. (2003). Guía para el monitoreo de la Meta 11 "Para el año 2020 haber Mejorado Sustancialmente la Vida de por lo Menos 100 Millones de Habitantes de Asentamientos Precarios". Recuperado de https://docs.google.com/document/ d/12J_-7kBsZ-0r07KqjzaGEQ43I1eG82-3g5CT-yhZM9l/ preview

Vidal, T., \& Pol, E. (2005). La apropiación del espacio: una propuesta teórica para comprender la vinculación entre las personas y los lugares. Anuario de Psicología, 36(3), 281 $-297$.

Winchester, L. (2006). El desarrollo sostenible de los asentamientos humanos en América Latina y el Caribe. Serie Medio ambiente y desarrollo. 